\title{
Decoherence in Josephson-junction qubits due to critical current fluctuations
}

\author{
D.J. Van Harlingen,${ }^{1}$ T.L. Robertson, ${ }^{2}$ B.L.T. Plourde,${ }^{2}$ P.A. Reichardt,${ }^{2}$ T.A. Crane,${ }^{1}$ and John Clarke ${ }^{2}$ \\ ${ }^{1}$ Department of Physics, University of Illinois at Urbana-Champaign, Urbana, IL 61801 \\ ${ }^{2}$ Department of Physics, University of California, Berkeley, CA 94720
}

(Dated: 12 December 2003)

\begin{abstract}
We compute the decoherence caused by $1 / f$ fluctuations at low frequency $f$ in the critical current $I_{0}$ of Josephson junctions incorporated into flux, phase, charge and hybrid flux-charge superconducting quantum bits (qubits). The dephasing time $\tau_{\phi}$ scales as $I_{0} / \Omega \Lambda S_{I_{0}}^{1 / 2}(1 \mathrm{~Hz})$, where $\Omega / 2 \pi$ is the energy level splitting frequency, $S_{I_{0}}(1 \mathrm{~Hz})$ is the spectral density of the critical current noise at 1 $\mathrm{Hz}$, and $\Lambda \equiv\left|I_{0} d \Omega / \Omega d I_{0}\right|$ is a parameter computed for given parameters for each type of qubit that specifies the sensitivity of the level splitting to critical current fluctuations. Computer simulations show that the envelope of the coherent oscillations of any qubit after time $t$ scales as $\exp \left(-t^{2} / 2 \tau_{\phi}^{2}\right)$ when the dephasing due to critical current noise dominates the dephasing from all sources of dissipation. We compile published results for fluctuations in the critical current of Josephson tunnel junctions fabricated with different technologies and a wide range in $I_{0}$ and $A$, and show that their values of $S_{I_{0}}(1 \mathrm{~Hz})$ scale to within a factor of three of $\left[144\left(I_{0} / \mu \mathrm{A}\right)^{2} /\left(A / \mu \mathrm{m}^{2}\right)\right](\mathrm{pA})^{2} / \mathrm{Hz}$ at $4.2 \mathrm{~K}$. We empirically extrapolate $S_{I_{0}}^{1 / 2}(1 \mathrm{~Hz})$ to lower temperatures using a scaling $T(\mathrm{~K}) / 4.2$. Using this result, we find that the predicted values of $\tau_{\phi}$ at $100 \mathrm{mK}$ range from 0.8 to $12 \mu \mathrm{s}$, and are usually substantially longer than values measured experimentally at lower temperatures.
\end{abstract}

PACS numbers: 85.25.Cp, 85.25.Am, 03.67.Lx

\section{INTRODUCTION}

Superconducting devices involving Josephson junctions are leading candidates for quantum bits (qubits) because of their manufacturability, controllability and scalability. Broadly speaking, there are three types of superconducting qubits. The first type is the flux qubit, which consists of a superconducting loop interrupted by either one ${ }^{1.2}$ or three $e^{3.4}$ junctions. When the qubit is biased at the degeneracy point the two states represented by magnetic flux pointing up and pointing down are superposed to produce symmetric and antisymmetric eigenstates. Quantum coherent behavior has been verified by means of spectroscopic measurements of the level splitting of these states 1.3 and by the observation of Rabi oscillations ${ }^{4}$ The second type of qubit is based on the charge degree of freedom, and consists of a nanoscale superconducting island coupled to a superconducting reservoir via a Josephson junction. The two quantum states differ by a single Cooper pair. Superpositions of these states have been demonstrated through Rabi oscillations,,$\frac{5}{=}$ and signatures of the entanglement of two charge qubits have been observed $\underline{\underline{6}}$ These two qubit types are distinguished by whether the Josephson coupling energy $E_{J}$ or the charging energy $E_{C}$ dominates the junction dynamics. A hybrid charge-flux device was operated in the crossover between these two regimes, at its degeneracy points in both charge and flux, ${ }^{7.8}$ it exhibited the longest dephasing time yet reported for a superconducting qubit, about $0.5 \mu \mathrm{s}$. The third type is the phase qubit, which consists of a single Josephson junction current-biased in the zero voltage state ${ }^{9.10}$ In this case, the two quantum states are the ground and first-excited states of the tilted potential well, between which Rabi oscillations have been observed. Unlike the other qubits, the phase qubit does not have a degeneracy point.

For all these qubits, the measured decoherence times are substantially shorter than predicted by the simplest models of decoherence from dissipative sources and than would be necessary for the operation of a quantum computer. As a result, there is an ongoing search to identify additional sources of dephasing. In the case of charge qubits, the coherence times have been limited by low frequency fluctuations of background charges in the substrate which couple capacitively to the island, thus dephasing the quantum state $\stackrel{11}{\underline{1}}$ Flux and phase qubits are essentially immune to fluctuations of charge in the substrate, and, by careful design and shielding, can also be made insensitive to flux noise generated by either the motion of vortices in the superconducting films or by external magnetic noise. The flux-charge hybrid, operated at its double degeneracy point, is intrinsically immune to both charge and flux fluctuations. However, all of these qubits remain sensitive to fluctuations in the critical current of the tunnel junctions at low frequency $f$, which lead to variations in the level splitting frequency over the course of the measurement and hence to dephasing.

Martinis et al $\stackrel{12}{\underline{12}}$ analyzed decoherence in phase qubits due to low frequency critical current fluctuations, and Paladino et al $\frac{13}{n}$ treated decoherence in charge qubits due to low frequency charge noise. In this paper, we explore the effects of low frequency noise in the critical current on the dephasing times $\tau_{\phi}$ in various superconducting qubits incorporating Josephson junctions, and compare our results with measured decoherence times. In Sec. II we discuss two sources of low frequency fluctuations in superconducting circuits and explain how they induce dephasing. In Sec. III we calculate the sensitivity of several Josephson qubit schemes to critical current variations, using parameters from recent experiments re- 
porting dephasing times. In Sec. IV we compile a list of measurements of the critical current noise in a variety of junctions and obtain a "universal value" that we use in subsequent estimates of decoherence times. In Sec. V we estimate dephasing times limited by $1 / f$ noise, using numerical simulations to elucidate the dephasing process. Section VI contains some concluding remarks.

\section{DECOHERENCE MECHANISM FOR LOW FREQUENCY NOISE}

We consider two intrinsic sources of low frequency noise in superconducting devices which can cause dephasing. Flux vortices hopping between pinning sites in superconducting films, illustrated in Fig. 1(a), result in fluctuations of the magnetic flux in multiply-connected superconducting circuits. Specifically, in superconducting flux qubits operating at the degeneracy of the left and right circulating current states, external magnetic flux $\Phi_{x}$ breaks the degeneracy, causing a second-order change in the tunneling frequency. This mechanism can usually be made negligible in devices fabricated with linewidths less than approximately $\left(\Phi_{0} / B\right)^{1 / 2}$ for which vortex trapping in the line is suppressed, ${ }^{14.15}$ here $\Phi_{0} \equiv h / 2 e$ is the flux quantum and $B$ is the field in which the device is cooled.

A more serious problem is critical current fluctuations caused by charge trapping at defect sites in the tunneling barrier, as in Fig. 1(b). In the prevailing picture, trapped charges block tunneling through a region of the junction due to the Coulomb repulsion, effectively modulating the junction area. In general, a single charge fluctuator produces a two-level, telegraph signal in the critical current of a junction, characterized by lifetimes in the untrapped (high critical current) state $\tau_{u}$ and the trapped (low critical current) state $\tau_{t}$. This produces a Lorentzian bump in the power spectral density with a characteristic time $\tau_{\text {eff }}=\left(1 / \tau_{t}+1 / \tau_{u}\right)^{-1}$. The dynamics of such fluctuators in junctions have been extensively studied ${ }^{16.17 .18}$, and the lifetimes have been measured as a function of temperature and voltage bias. There is strong evidence from the voltage dependence that the dominant charges enter the barrier from one electrode and exit to the other, and that the fluctuators exhibit a crossover from thermal activation to tunneling behavior at about $15 \mathrm{~K}$. In the tunneling regime, the fluctuating entity has been shown to involve an atomic mass, suggesting that ionic reconfiguration plays an important role in the tunneling process. Interactions between traps resulting in multiple level hierarchical kinetics have been observed,, 19 but usually the traps can be considered to be local and non-interacting. In this limit, the coexisting traps produce a distribution of Lorentzian features that superimpose to give a $1 / f$-like spectrum. 20.21

The parametric fluctuations in the qubit energy levels introduce phase noise into the measurement of the probability distribution of the qubit states. The key point
FIG. 1: note: figure attached Effects of low frequency flux and critical current fluctuations in a superconducting qubit. (a) Flux modulation from vortices hopping into and out of a loop, and critical current modulation from electrons $e^{-}$temporarily trapped at defect sites in the junction barrier. (b) A single charge trap blocks tunneling over an area $\delta A$, reducing the critical current. (c) Fluctuations modify the oscillation frequency, inducing phase noise which leads to decoherence in time-averaged ensembles of sequential measurements of the qubit observable $Z$.

is that determination of the qubit state and its evolution with time requires a large number of measurements. In the presence of low frequency noise, the energy levels fluctuate during the data acquisition. This causes an effective decoherence in the qubit, as illustrated in Fig. 11(c). The resulting decay of the qubit state probability amplitude reflects the spectrum of the low frequency noise.

\section{QUBIT SENSITIVITY TO CRITICAL CURRENT FLUCTUATIONS}

We consider a superconducting qubit with quantum states separated in energy by $\hbar \Omega$, and assume that the splitting depends on the critical current of one or more Josephson tunnel junctions in the qubit. The sensitivity of the energy difference to critical current fluctuations is described by the dimensionless parameter

$$
\Lambda=\left|I_{0} d \Omega / \Omega d I_{0}\right|
$$

the fractional change in the energy separation for a given fractional change in the critical current $I_{0}$. The value of $\Lambda$ depends on the qubit architecture, the device parameters, and the bias point. A large value of $\Lambda$ indicates that a particular qubit type is vulnerable to decoherence caused by critical current fluctuations; small values indicate a more robust qubit design for fluctuations of the same amplitude. In the following sections, we calculate $\Lambda$ for a variety of qubit designs and parameters used in recent experiments. In some cases, we can develop analytical expressions for the energy separation, which often is a tunneling matrix element, from which $\Lambda$ can be calculated; in others, it is necessary to carry out numerical calculations to estimate the response to critical current changes.

\section{A. One-Junction Flux Qubit (Ground State)}

We first consider the one-junction flux qubit [Fig. 2(a)], consisting of a single Josephson junction of critical current $I_{0}$ and capacitance $C$ in a loop of inductance $L$ biased with an applied flux $\Phi_{x}$. At the degeneracy point $\Phi_{x}=\Phi_{0} / 2$, the energy vs. flux curve is a degenerate double-well potential given by $V(\phi)=$ 
FIG. 2: note: figure attached One-junction flux qubit. (a) Schematic. (b) Symmetric double well potential for flux bias $\Phi_{x}=\Phi_{0} / 2$. (c) Flux fluctuation $\Delta \Phi$ couples to $\Omega$ only in second order. (d) Critical current fluctuation $\Delta I_{0}$ produces exponential change in $\Omega$.

$\left(\Phi_{0}^{2} / 8 \pi^{2} L\right)\left[2 \beta_{L} \cos (\phi)+\left(\phi+\pi+2 \pi \Phi_{x} / \Phi_{0}\right)^{2}\right]$, in terms of the junction phase $\phi$. The two states of lowest energy are approximately symmetric and antisymmetric combinations of localized states in the left and right wells characterized by clockwise and counterclockwise circulating currents, between which the "phase particle" tunnels [Fig. 2(b)]. Fluctuations in the flux tilt the potential wells, weakly changing the tunneling frequency in second order [Fig. 2] (c)]; however, critical current fluctuations directly modulate the barrier height, producing an exponential change in the qubit tunneling frequency [Fig. 2(d)].

We now calculate the tunnel splitting, or more precisely the energy difference between the ground and first excited state, for the one-junction flux qubit using three different methods. The purpose of this pedagogical exercise is to understand in which regimes certain approximations are valid. We build on this insight to analyze other qubits later in this paper.

Our first approach is to approximate the potential with a quartic polynomial and quote an analytic result for the tunneling frequency in the semi-classical WKB approximation, $\frac{2}{2}$

$$
\Omega=\omega_{0} \exp \left[-\eta\left(\beta_{L}-1\right)^{3 / 2}\right] .
$$

Here $\omega_{0} \equiv 2\left[\left(\beta_{L}-1\right) / L C\right]^{1 / 2}$ is the classical frequency of small oscillations in the bottom of the wells, $\beta_{L} \equiv$ $2 \pi L I_{0} / \Phi_{0}$ is the dimensionless screening parameter, and $\eta \equiv\left(8 I_{0} C \Phi_{0}^{3} / \pi^{3} \hbar^{2}\right)^{1 / 2}$ is a parameter which describes the "degree of classicality" and hence determines when quantum tunneling is important..$^{2}$ Figure 3 (a) plots $\Omega / 2 \pi$ vs. $\beta_{L}$ for stated values of $L$ and $C$.

However, the semi-classical approximation is valid only in the regime where the bound states in each well nearly form a continuum, which is far from the case we consider here with only one bound state in each well. To obtain the correct splittings for the ground state in the WKB approximation one must modify Eq. (2). A more accurate result is ${ }^{22}$

$$
\Omega=2 \omega_{0} \sqrt{\frac{m \omega_{0} \phi_{m}^{2}}{\pi \hbar}} e^{A} e^{-S_{0} / \hbar},
$$

where $S_{0}$ is the action along the tunneling direction

$$
S_{0}=\int_{-\phi_{m}}^{\phi_{m}} \sqrt{2 m V(\phi)} d \phi
$$

and $A$ is a correction factor

$$
A=\int_{0}^{\phi_{m}}\left[\frac{m \omega_{0}}{\sqrt{2 m V(\phi)}}-\frac{1}{\phi_{m}-\phi}\right] d \phi .
$$

Here $m=C\left(\Phi_{0} / 2 \pi\right)^{2}$ is the effective mass of the tunneling particle, and $\pm \phi_{m}$ are the positions of the minima of the symmetric double well potential. The great advantage of this formulation of the WKB approximation, beyond its validity for ground state splittings, is that the limits of the integrals are at the true extrema of the potential rather than the classical turning points, making the calculation more tractable.

By evaluating Eqs. (3)-(5) numerically, we obtain a second result for $\Omega$, shown in Fig. 3(a) as a function of $\beta_{L}$. We see that the two forms of the WKB approximation are similar in overall shape, with $\Omega$ vanishing at $\beta_{L}=1$ where $\omega_{0}$ becomes zero, and decreasing exponentially at larger values of $\beta_{L}$. However, the two forms disagree quantitatively at small values of $\beta_{L}$ and diverge from one another at large values of $\beta_{L}$. These difficulties are hardly surprising, since the WKB approximation assumes a well-defined state localized in each well, and for states very close to the top of the barrier this assumption is no longer valid. Thus, to obtain a more accurate tunneling frequency we need a full quantum mechanical solution for the degenerate double-well potential.

To find the wavefunctions we first choose a set of basis functions $b_{i}(\phi)$. By calculating the Hamiltonian matrix elements

$$
H_{m n}=\int_{-\infty}^{\infty} b_{n}(\phi) H(\phi) b_{m}(\phi) d \phi
$$

and the overlap matrix

$$
B_{m n}=\int_{-\infty}^{\infty} b_{n}(\phi) b_{m}(\phi) d \phi,
$$

we can find the energy levels as the eigenvalues of the matrix

$$
K=B^{-1} H
$$

To solve for the ground state wavefunction we choose as our basis set 12 simple harmonic oscillator wavefunctions centered in the left well and 12 more centered in the right well. We use the Hamiltonian

$$
\begin{aligned}
H(\phi)= & \frac{\Phi_{0}^{2}}{8 \pi^{2} L}\left[2 \beta_{L} \cos (\phi)+\left(\pi+\phi+\phi_{x}\right)^{2}\right] \\
& +\frac{\Phi_{0}^{2} C}{8 \pi^{2}}\left(\frac{\partial}{\partial \phi}\right)^{2}
\end{aligned}
$$

where $\phi_{x} \equiv 2 \pi \Phi_{x} / \Phi_{0}$. The results for $\phi_{x}=0$ are shown in Fig. 3(a). For large values of $\beta_{L}$ the full solution approaches the modified WKB expression, Eq. (3), asymptotically. As $\beta_{L}$ is decreased toward unity the tunneling rate approaches a constant value. This is in contrast to the semi-classical models which predict a tunneling rate proportional to $\beta_{L}$ as the prefactor $\omega_{0}$ dominates; the full solution shows that this is an artifact of the approximation.

Figure 3(b) shows $\Lambda$ vs. $\beta_{L}$ for the three calculations. The two semi-classical approximations predict that $\Lambda$ 


\section{FIG. 3: note: figure attached}

Three quantities for the ground state of the one-junction flux qubit at the degeneracy point calculated using the standard WKB approximation (solid), WKB approximation corrected for the ground state (dashed), and numerical solution for the wavefunctions (points), plotted as a function of the dimensionless screening parameter $\beta_{L}$. (a) Splitting frequency between ground and first excited states, (b) sensitivity parameter $\Lambda$, and (c) effects of critical current fluctuations of three magnitudes on tunneling rate.

Parameters are from Friedman et al.: $L=240 \mathrm{pH}$ and

$$
C=104 \mathrm{fF}, 1
$$

vanishes at certain values of $\beta_{L}$, but this is an artifact of the apparent maxima in Fig. 31(a); the full quantum treatment shows no zero. Figure 3 (c) plots the fractional change in tunneling frequency, $\delta \Omega / \Omega$, vs. $\beta_{L}$ for the three calculations for three fractional changes in critical current, $\delta I_{0} / I_{0}$. We note that for $\beta_{L} \gtrsim 1.1$ the three approaches differ by no more than a factor of about two.

\section{B. One-Junction Flux Qubit (Excited States)}

The first demonstration of a one-junction flux qubit did not employ ground states, however, but excited states in deep, tilted potential wells $\frac{1}{2}$ The WKB approximation is again unsuitable, for two main reasons. First, treating asymmetric potentials is more difficult, because of different prefactors for the two wells, but this can be overcome ${ }^{23}$ More importantly, resonant tunneling, which causes a dramatic increase in the tunneling rate when two energy levels are aligned, is entirely absent from the WKB approximation. Thus, the only way to calculate the sensitivity to critical current fluctuations is to solve the Schrödinger equation for the energy levels numerically.

We adopt the approach of Sec. IV.A with a different basis set. We use 60 harmonic oscillator wavefunctions centered between the minima of the two wells, so that $B$ becomes the identity matrix. To reproduce the experimental conditions, $\frac{1}{-}$ we set $\beta_{L}=1.5$ and find the energy levels for successive values of applied flux $\phi_{x}$. We find that the energy difference between the third and ninth excited states has a local minimum at $\phi_{x} \approx 0.514 \times 2 \pi$, corresponding to the condition for resonant tunneling. Fixing $\phi_{x}$ at this value and sweeping $\beta_{L}$, we calculate the relevant quantities for low frequency critical current fluctuations. The results are shown in Fig. 4.

In Fig. 4(a) we see that near the resonant point $\beta_{L}=1.5, \Omega$ decreases with increasing barrier height, as one would expect from a semi-classical analysis, but reaches a local minimum at a slightly higher value. As $\beta_{L}$ is increased further, $\Omega$ increases because the energy levels are no longer resonant. At the minimum, the derivative quantity $\Lambda$ vanishes, as the changing barrier height balances the loss of resonance, indicating that the system is immune to small critical current fluctuations at this

\section{FIG. 4: note: figure attached}

Numerical solution for the excited states of an asymmetric one-junction flux qubit. (a) Tunneling frequency between the third excited state in the shallow well and the ninth excited state in the deep well as a function of $\beta_{L}$ for a system on resonance at $\beta_{L}=1.5$. (b) Derived sensitivity to critical current fluctuations. Device parameters are as in Fig. 3]

point. We note that on resonance, where $\Lambda$ is almost optimally bad, the system is immune to flux noise, because the energy is a minimum as a function of flux. Thus, one can exchange sensitivity to critical current fluctuations for sensitivity to flux noise as appropriate.

\section{Three-junction flux qubit}

The three-junction qubit consists of three Josephson junctions of critical currents $I_{0}^{a}, I_{0}^{b}$, and $I_{0}^{c}$ in series in a superconducting loop of geometric inductance $L$, as shown in Fig. 5(a) 3.4.24 The smallest of the junctions, $c$, primarily controls the barrier height while the larger two junctions, $a$ and $b$, serve as Josephson inductors. We parameterize this device by the ratios of the Josephson coupling energy of the three junctions to the charging energy $E_{C}=e^{2} / 2 C$, where $C$ is the mean capacitance of the two larger junctions: $E_{J}^{a, b, c} / E_{C}=I_{0}^{a, b, c} \Phi_{0} / 2 \pi E_{C}=\gamma^{a, b, c}$. We assume that the junctions are in the phase regime where $\gamma^{a, b, c}>>1$ and require that $1 / 2<2 \gamma^{c} /\left(\gamma^{a}+\gamma^{b}\right)<$ 1 so that a double-well potential is formed. We consider the junctions individually so that we may allow their critical currents to fluctuate independently, and consider the case where asymmetries in the large junctions are small, i.e. $2 \gamma^{b} /\left(\gamma^{a}+\gamma^{b}\right)<<1$. The energy landscape at applied flux $\Phi_{0} / 2$ exhibits multiple wells, most notably two degenerate wells separated by a tunnel barrier that is much lower than the barriers to all other flux states. The potential can be written

$$
V(\delta)=\left(E_{C} / 8 C\right)\left(\gamma^{a}+\gamma^{b}+4 \gamma^{c} \cos \delta\right)^{2},
$$

where $\delta$ is a variable aligned with the tunneling direction that is derived from the three junction phases. In the small-inductance limit, we can apply the WKB approximation given in Eqs. (3)-(5) to calculate the rate for this so-called intracell tunneling 


$$
\Omega=\frac{\Gamma E_{C}}{\hbar} \exp \left[-\frac{\left(4 \gamma^{c}+\gamma^{a}+\gamma^{b}\right)\left\{\sqrt{\left(4 \gamma^{c}\right)^{2}-\left(\gamma^{a}+\gamma^{b}\right)^{2}}-\left(\gamma^{a}+\gamma^{b}\right) \arccos \left(\frac{\gamma^{a}+\gamma^{b}}{4 \gamma^{c}}\right)\right\}}{2 \sqrt{\gamma^{c}\left(\gamma^{a}+\gamma^{b}\right)\left(4 \gamma^{c}+\gamma^{a}+\gamma^{b}\right)}}\right]
$$

where

$$
\Gamma=\frac{\left(4 \gamma^{c}-\gamma^{a}-\gamma^{b}\right)^{5 / 4}\left(\gamma^{a}+\gamma^{b}\right)^{1 / 4}\left(4 \gamma^{c}+\gamma^{a}+\gamma^{b}\right)}{2 \pi^{1 / 2}\left(\gamma^{c}\right)^{7 / 4}}
$$

FIG. 5: note: figure attached Three-junction flux qubit. (a) Schematic showing inductive loop, embracing $\Phi_{0} / 2$ interrupted by three Josephson junctions. (b) Tunneling frequency and (c) $\Lambda$ vs. Josephson-to-charging energy ratio. Solid lines indicate dependence on large junction ratio $\gamma^{a, b}$ with $\gamma^{c}=28$, and dashed lines indicate dependence on small junction ratio $\gamma^{c}$ with $\gamma^{a}=\gamma^{b}=35$. $E_{C}=7.4 \mathrm{GHz}$ for all plots.

We note that the exponent reduces to a form previously obtained $^{24}$ when $\gamma^{a}=\gamma^{b}$; however the prefactor differs.

To calculate the effects of low frequency noise, we must account for the fact that the critical currents of the three junctions fluctuate independently. Because the small and large junctions play different roles, we consider changes in each separately. We adopt parameters used in the experiments of Chiorescu et al. $\frac{4}{\underline{4}} \gamma^{a}=\gamma^{b}=35$, $\gamma^{c}=0.8 \times \gamma^{a, b}=28$, and $E_{C} / 2 \pi \hbar=7.4 \mathrm{GHz}$. In Fig. 5 (b), we plot the tunneling frequency $\Omega / 2 \pi$ as a function of the Josephson-to-charging energy ratios for each of the three junctions holding the other two constant. Figure 5(c) shows $\Lambda_{i}=\left(\gamma^{i} / \Omega\right) \partial \Omega / \partial \gamma^{i}$, where $i=a, b$ or $c$, as a function of the same variables. For the experimental parameters, we calculate $\Omega / 2 \pi=7.96 \mathrm{GHz}$, which differs somewhat from the experimentally observed value of 3.4 $\mathrm{GHz}$; however the exponential dependence in Eq. (11) magnifies parametric uncertainties, making exact agreement unlikely. We see that the small junction is indeed the dominant contribution to $\Lambda$, with $\Lambda_{a, b}=4.6$ and $\Lambda_{c}=10.4$. Adding the contributions incoherently gives $\Lambda=\left(\Lambda_{a}^{2}+\Lambda_{b}^{2}+\Lambda_{c}^{2}\right)^{1 / 2}=12.3$.

\section{Single Josephson junction (phase) qubit}

Martinis and coworkers have used a single, currentbiased Josephson junction as a qubit, the $|0\rangle$ and $|1\rangle$ states being the ground and first excited states of the tilted washboard potential well, as shown in Fig. 6(a). The energy separation between energies $E_{0}$ and $E_{1}$ is

$$
\Omega=\left(E_{1}-E_{0}\right) / \hbar \approx \omega_{p},
$$

where

$$
\omega_{p}=\left(2 \sqrt{2} \pi I_{0} / C \Phi_{0}\right)^{1 / 2}\left(1-I / I_{0}\right)^{1 / 4}
$$

FIG. 6: note: figure attached Single Josephson junction qubit. (a) Schematic and (b) energy level diagram. (c) Variation of energy separation with bias current. (d) $\Lambda$ as a function of bias current. Parameters are from Martinis et al: $C=6 \mathrm{pF}$, corresponding to a junction area of $100 \mu \mathrm{m}^{2}$, and $I_{0}=21.1$ $\mu \mathrm{A} ?^{9}$

is the small oscillation (plasma) frequency in the well. In Fig. 6(b) we plot $\Omega$ vs. $I / I_{0}$ for the parameters used in the experiments of Martinis et al. .9 We determine $\Lambda$ vs. $I / I_{0}$ from Eq. (14), and plot the result in Fig. 6(c). At the bias point used in the experiments, $I=20.77$ $\mu \mathrm{A}\left(I / I_{0}=0.985\right), \Lambda$ has the value 16 at a tunneling frequency $\Omega / 2 \pi=6.9 \mathrm{GHz}$.

\section{E. Quantronium (hybrid charge-flux) qubit}

The qubit developed by the Saclay group consists of a Cooper pair box, a small island coupled by Josephson junctions of critical current $I_{0}$ and capacitance $C_{j}$ on each side, connected in a superconducting loop containing a Josephson junction with a much larger critical current [Fig. [7(a)] $]^{\underline{7}}$ The island is connected to a voltage source via a capacitor $C_{g}$. The circuit parameters are selected with the Josephson energy $E_{J}^{a, b}=\Phi_{0} I_{0}^{a, b} / 2 \pi$ comparable to the charging energy $E_{C P}=(2 e)^{2} / 2\left(C_{g}+2 C_{j}\right)$, so that the device operates in the crossover regime between the charge and flux modes. In this configuration, a charge induced on the central island generates a phase change around the loop, driving a circulating current determined by the Josephson inductance of the two small junctions. This current is detected by measuring the pulsed current required to exceed the critical current of the readout junction, $I_{0}^{r}$. The qubit energy levels $E_{0}$ and $E_{1}$ are controlled by the gate charge $N_{g} e$ and the phase difference $\delta$ across both junctions according to the approximation ${ }^{8}$

$$
E_{0,1}=\mp\left\{\left[\frac{E_{J}}{2} \cos \left(\frac{\delta}{2}\right)\right]^{2}+\left[E_{C P}\left(1-2 N_{g}\right)\right]^{2}\right\}^{1 / 2}(15)
$$

where $E_{J}=E_{J}^{a}+E_{J}^{b}$ is the total Josephson coupling energy. Thus, the qubit frequency, which is proportional 
FIG. 7: note: figure attached The quantronium qubit, which operates in the crossover regime between the charge and flux modes, converts charge oscillations on the single electron transistor to flux modulation in the loop. (a) Schematic showing phase difference $\delta$ across two small Josephson junctions with charge $N_{g}$ on island between them. (b) Level splitting frequency $\Omega / 2 \pi$ and (c) critical current sensitivity $\Lambda$ vs. $N_{g}$. Curves are plotted for the parameters reported by Vion et al., $I_{0}=18 \mathrm{nA}, C_{j}=2.7 \mathrm{fF}$; at the optimal working point $N_{g}=1 / 2, \delta=0, \Lambda=2^{-1 / 2}$, and $\Omega$ is calculated to be 17.9 $\mathrm{GHz}$, slightly different from the observed value of $16.5 \mathrm{GHz}$.

to the level spacing, is

$$
\begin{aligned}
\hbar \Omega & =E_{1}-E_{0} \\
& =2\left\{\left[\frac{E_{J}}{2} \cos \left(\frac{\delta}{2}\right)\right]^{2}+\left[E_{C P}\left(1-2 N_{g}\right)\right]^{2}\right\}^{1 / 2}
\end{aligned}
$$

When $N_{g}$ and $\delta$ are adjusted to the optimal working point, $\delta=0$ and $N_{g}=1 / 2$, the system is maximally insensitive to phase and charge fluctuations; however, incoherent fluctuations in the critical current of the small junctions couple linearly to the level splitting without perturbing the phase or charge to first order, giving $\Lambda=2^{-1 / 2}$. Away from $N_{g}=1 / 2, \Lambda$ is reduced, as plotted in Fig. Z(b) for the parameters used in the Saclay experiments, $C_{j}=2.7 \mathrm{fF}\left(E_{C P} / k_{B}=0.68 \mathrm{~K}\right)$, and $I_{0}=18$ $\mathrm{nA}\left[\left(E_{J}^{a}+E_{J}^{b}\right) / k_{B}=0.86 \mathrm{~K}\right]$, but the device is then no longer immune to charge fluctuations.

\section{1/F CRITICAL CURRENT FLUCTUATIONS}

Critical current fluctuations in Josephson junctions have been extensively studied over the past two decades, mostly to understand the low frequency noise in SQUIDs. As a result, most of the reported measurements have been in the temperature range $1-4 \mathrm{~K}$ on junctions of areas from $4-100 \mu \mathrm{m}^{2}$. We first briefly describe scaling of the data by the junction area, the critical current, and temperature.

As mentioned earlier, it is generally accepted that critical current noise in Josephson junctions arises from charge trapping at defect sites in the barrier. A trapped charge locally modifies the height of the tunnel barrier, changing the resistance of the junction, and, in the case of a Josephson junction, also the critical current. For a junction of area $\mathrm{A}$, the change in critical current is $\Delta I_{0}=(\Delta A / A) I_{0}$, where $\Delta A$ is the effective area of the junction over which tunneling is blocked by the temporary presence of the trapped charge. The critical current spectral density for one trap is proportional to $\left(\Delta I_{0}\right)^{2}$, so that the spectral density for $N$ identical, independent traps scales as $N\left(\Delta I_{0}\right)^{2}=n A(\Delta A / A)^{2} I_{0}{ }^{2}$, where $n$ is the number of traps per unit area. Consequently, for a given junction technology characterized by a trap density $n$ and blocking area $\delta A$, we expect the critical current spectral density $S_{I_{0}}(f)$ to scale as $I_{0}^{2} / A$. To test this hypothesis, we have compiled a series of measurements of the $1 / f$ critical current noise at temperature $T=4.2 \mathrm{~K}$, taken in a variety of junctions and dc SQUIDs by different groups (Table I). For each, we list the critical current $I_{0}$ and area $A$ of the junctions, which vary by several orders of magnitude, and the magnitude of the critical current noise spectral density at $1 \mathrm{~Hz}, S_{I_{0}}(1 \mathrm{~Hz})$. We observe that the quantity $S_{I_{0}}^{1 / 2}(1 \mathrm{~Hz}) A^{1 / 2} / I_{0}$ is remarkably constant, varying by less than a factor of 3 .

This result supports the charge trap model for the $1 / f$ critical current noise, and, since it includes measurements on different junction barrier materials $(\mathrm{AlOx}$, $\mathrm{InOx}, \mathrm{NbOx}$ ) even suggests that the product of the trap density and Coulomb screening area must be similar in magnitude for these different oxides.

Averaging these measurements, we estimate the critical current noise at $4.2 \mathrm{~K}$ for any junction of critical current $I_{0}$ and area $A$ to be

$$
S_{I_{0}}(1 \mathrm{~Hz}, 4.2 \mathrm{~K}) \approx 144 \frac{\left(I_{0} / \mu \mathrm{A}\right)^{2}}{A / \mu \mathrm{m}^{2}} \frac{(\mathrm{pA})^{2}}{\mathrm{~Hz}} .
$$

The temperature dependence of the $1 / f$ critical current noise is less firmly established. Since the charge traps responsible for the noise are thought to be in the tunneling regime at low temperatures, one might expect that the temperature dependence would be weak. However, the only measurement of the spectral density of the critical current noise in Josephson junctions at low temperatures we are aware of showed a $T^{2}$ dependence from $4.2 \mathrm{~K}$ down to about $300 \mathrm{mK} 25$ The issue of whether or not this behavior extends to lower temperatures is of crucial importance to the development of qubits involving Josephson junctions.

In the absence of other data or models, we take the optimistic view that $S_{I_{0}}(f, T)$ scales quadratically with temperature and so is dramatically reduced at the low temperatures where superconducting qubits are operated. We thus take as a working hypothesis

$$
S_{I_{0}}(f, T) \approx\left[144 \frac{\left(I_{0} / \mu \mathrm{A}\right)^{2}}{\left(A / \mu \mathrm{m}^{2}\right)}\left(\frac{T}{4.2 \mathrm{~K}}\right)^{2}(\mathrm{pA})^{2}\right] \frac{1}{f} .
$$

The observed $T^{2}$ dependence is incompatible with the electron trapping mechanism in the tunneling regime, which predicts a linear temperature dependence ${ }^{20}$ There is strong evidence that charge trapping occurs via tunneling in the temperature range considered, so that the noise should be relatively temperature independent. Further, for $e V, k_{B} T<<2 \Delta$, where $\Delta$ is the energy gap, both the available number of single electrons and the available number of final single-electron states scale as $\exp \left(-\Delta / k_{B} T\right)$, so that charge trapping is expected to freeze out at low temperatures. This leads one to seek alternative explanations. One possibility is that the $1 / f$ noise is associated with leakage currents at voltages below $2 \Delta / e$, which do not exhibit an exponential tempera- 
ture dependence. Such leakage currents presumably occur between opposing normal regions of the electrodes, conceivably at the edges of the junctions or along the core of a flux vortex penetrating the junction. An investigation of the correlation between leakage currents and $1 / f$ noise would be of great interest. Other possible sources of the $1 / f$ noise include the motion of electrons between traps within the tunnel barrier, and the motion of vortices in or near the junction, which could create a thermally-activated contribution to the critical current fluctuations. We note that a thermally activated model yielding a $T^{2}$ dependence has been proposed by Kenyon et $a l^{26}$ in the context of charge $1 / f$ noise, but should be equally applicable to critical current noise. In this model, one assumes that the two-state systems have asymmetric wells, and that the depths of the wells are independent random variables.

\section{DETERMINATION OF DEPHASING TIMES}

As described above, the low frequency critical current fluctuations generate phase noise and decoherence in any measurement of quantum coherent oscillations. To determine the effect of the fluctuations on $\tau_{\phi}$, we simulate the oscillations of the qubit state probability distribution.

In general, there are two techniques for observing quantum oscillations in superconducting qubits. The qubit bias can be pulsed suddenly to the degeneracy point where the qubit oscillates between the measurement basis states at frequency $\Omega$. After time $t$, the qubit bias is pulsed suddenly away from the degeneracy point, af-

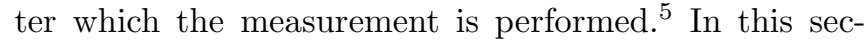
tion we consider such a degeneracy point measurement for a superconducting qubit in the presence of low frequency critical current fluctuations. We normalize the qubit states to +1 and -1 and always initialize the state to +1 before each bias pulse to the degeneracy point. For qubits coupled to Ohmic dissipation and without critical current fluctuations, the subsequent oscillations of the expectation value $\langle Z(t)\rangle$ decay with the dephasing time $\tau_{\phi}^{0}$ according to

$$
\langle Z(t)\rangle=e^{-t / \tau_{\phi}^{0}} \cos \Omega t
$$

We will see that the low frequency noise provides an additional mechanism for decoherence and a different functional form for the decay of $\langle Z(t)\rangle$.

Alternatively the qubit bias can remain fixed away from the degeneracy point while the qubit is driven between the ground and excited states with resonant microwave pulses of varying width. This technique has been used to measure Rabi oscillations of the quantum state in several superconducting qubits $\stackrel{4.7 .9}{ }$ A measurement of the dephasing time $\tau_{\phi}$ in this driven case requires a more sophisticated pulse arrangement, such as a Ramsey fringe sequence $\stackrel{4.7}{=}$ We note that for the single Josephson junction phase qubit,,$\underline{9}$ resonant microwave driving is the only possible technique for observing quantum oscillations as there is no degeneracy point at which the qubit can be operated. Nonetheless, we expect our calculation of the dephasing due to critical current fluctuations from a simulation of an experiment involving switching to and away from the degeneracy point to give a reasonable estimate for $\tau_{\phi}$ in the microwave-driven experiments as well.

For our simulations of the quantum oscillations at the degeneracy point, we allow the qubit to evolve for time $t$ followed by a single-shot measurement with a sampling window that is much shorter than $2 \pi / \Omega$ (Fig. 8). We assume that the interval between consecutive single-shot measurements of the state is $t_{Z}$; this interval includes the time to initialize the state, the delay time during which the qubit evolves, the sampling time, the readout time, and any time allotted for the system to thermalize following the dissipative measurement. To map out the time dependence of the qubit state, we measure the expectation value $N_{t}$ times, at intervals separated by time $t_{d}$, each point being the average of $N_{Z}$ measurements. From this time evolution, we can determine the envelope and its characteristic decay time, and, if the sampling frequency is above the Nyquist frequency (twice the coherent oscillation frequency), the oscillation frequency. The key point is that low frequency fluctuations in the critical current cause the oscillation frequency to be different for each successive single-shot measurement of the qubit, resulting in an effective dephasing.

Because of the nature of $1 / f$ noise, the resulting dephasing depends both on the total number of samples $N=N_{Z} N_{t}$ (which sets the elapsed time of the experiment $N t_{Z}$ ) and on the sequence in which the measurements are taken. We consider two cases, illustrated in Fig. 8 Method A is time-delay averaging, in which we take $N_{Z}$ successive measurements for each time delay and average them to find the qubit expectation value at that delay time. Method B is time-sweep averaging, in which we make a single measurement at each of the $N_{t}$ points, and then average $N_{Z}$ such time sweeps to generate the qubit time evolution. These differ because of the time scales involved in $1 / f$ noise: Method A averages only high frequency fluctuations at each time-delay point, while Method B averages both high and low frequency components. Data sampling schemes intermediate between these extremes are also possible; these involve the averaging of $N_{s}<N_{Z}$ multiple sweeps, each acquired by sampling $N_{m}=N_{Z} / N_{s}$ successive measurements at each time delay value.

For method $\mathrm{A}$, the expectation value after time $t_{m}=$

FIG. 8: note: figure attached Measurement sequences for mapping out coherent oscillations. (a) Method A: time-delay averaging. (b) Method B: time-sweep averaging. The interval between qubit state measurements is $t_{Z}$; the spacing of time-delay points is $t_{d}$. 
TABLE I: Compilation of $1 / f$ critical current noise measurements in Josephson junctions of different technologies, areas $A$, and critical currents $I_{0}$ at $4.2 \mathrm{~K} ; S_{I_{0}}(1 \mathrm{~Hz})$ is the spectral density at $1 \mathrm{~Hz}$. The relative invariance of the scaled quantity $A^{1 / 2} S_{I_{0}}^{1 / 2}(1$ $\mathrm{Hz}) / I_{0}$ supports the charge trapping mechanism for the $1 / f$ noise.

\begin{tabular}{|c|c|c|c|c|}
\hline $\begin{array}{c}\text { Junction } \\
\text { technology }\end{array}$ & $\begin{array}{c}A \\
\mu \mathrm{m}^{2} \\
\end{array}$ & $\begin{array}{c}I_{0} \\
\mu A \\
\end{array}$ & $\begin{array}{l}S_{I_{0}}^{1 / 2}(1 \mathrm{~Hz}) \\
\mathrm{pA} / \mathrm{Hz}^{1 / 2}\end{array}$ & $\begin{array}{l}A^{1 / 2} S_{I_{0}}^{1 / 2}(1 \mathrm{~Hz}) / I_{0} \\
\mu \mathrm{m}\left(\mathrm{pA} / \mathrm{Hz}^{1 / 2}\right) / \mu \mathrm{A}\end{array}$ \\
\hline \multirow[t]{4}{*}{$\mathrm{Nb}-\mathrm{AlOx}-\mathrm{Nb}^{27}$} & 9 & 9.6 & 36 & 11 \\
\hline & 8 & 2.6 & 6 & 7 \\
\hline & 115 & 48 & 35 & 8 \\
\hline & 34 & 12 & 41 & 20 \\
\hline \multirow[t]{5}{*}{$\mathrm{Nb}-\mathrm{NbOx}-\mathrm{PbIn}^{25}$} & 4 & 21 & 74 & 7 \\
\hline & 4 & 4.6 & 46 & 20 \\
\hline & 4 & 5.5 & 25 & 9 \\
\hline & 4 & 5.7 & 34 & 12 \\
\hline & 4 & 11.4 & 91 & 16 \\
\hline $\mathrm{Nb}-\mathrm{NbOx}-\mathrm{PbInAu} \mathrm{u}^{28}$ & 1.8 & 30 & 184 & 8 \\
\hline $\mathrm{PbIn}-\mathrm{InOx}-\mathrm{Pb}^{29}$ & 6 & 510 & 3300 & 15 \\
\hline Average & & & & 12 \\
\hline
\end{tabular}

$m t_{d}$, with $1 \leq m \leq N_{t}$, is given by

$$
\begin{aligned}
\left\langle Z^{A}\left(t_{m}\right)\right\rangle & =\frac{1}{N_{Z}} \sum_{n=1}^{N_{Z}} \cos \left\{\left[\Omega+\frac{d \Omega}{d I_{0}} \delta I_{0}\left(t_{A}\right)\right] t_{m}\right\} e^{-\frac{t_{m}}{\tau_{\phi}^{0}}} \\
& =\frac{1}{N_{Z}} \sum_{n=1}^{N_{Z}} \cos \left\{\Omega\left[1+\Lambda \delta i_{0}\left(t_{A}\right)\right] t_{m}\right\} e^{-\frac{t_{m}}{\tau_{\phi}^{0}}}
\end{aligned}
$$

where $t_{A}=\left[(m-1) N_{Z}+n\right] t_{Z}$. For method B we have

$$
\begin{aligned}
\left\langle Z^{B}\left(t_{m}\right)\right\rangle & =\frac{1}{N_{Z}} \sum_{n=1}^{N_{Z}} \cos \left\{\left[\Omega+\frac{d \Omega}{d I_{0}} \delta I_{0}\left(t_{B}\right)\right] t_{m}\right\} e^{-\frac{t_{m}}{\tau_{\phi}^{0}}} \\
& =\frac{1}{N_{Z}} \sum_{n=1}^{N_{Z}} \cos \left\{\Omega\left[1+\Lambda \delta i_{0}\left(t_{B}\right)\right] t_{m}\right\} e^{-\frac{t_{m}}{\tau_{\phi}^{0}}}
\end{aligned}
$$

where $t_{B}=\left[(n-1) N_{t}+m\right] t_{Z}$. Here $\tau_{\phi}^{0}$ is the dephasing time set by decoherence mechanisms besides $1 / f$ noise such as dissipative processes in the qubit and the environment. To simulate the dephasing due to critical current fluctuations alone, we take $\tau_{\phi}^{0}$ to be infinite. The quantity $\delta I_{0}(t)$ is the time-varying deviation in the critical current from its average value. Note that the changes in oscillation frequency scale with $\Lambda$ and with the fractional changes in the critical current $\delta i_{0}(t)=\delta I_{0}(t) / I_{0}$.

We determine the time sequence of critical current fluctuations (Fig. 9) by Fourier transforming a complex spectrum of critical current fluctuations. This spectrum is generated in frequency space, with magnitudes randomly chosen from an exponential distribution with a mean value equal to $\left(S_{I_{0}}(1 \mathrm{~Hz}) / f\right)^{1 / 2}$ and phases randomly chosen from a uniform distribution from 0 to $2 \pi$.
FIG. 9: (note: figure attached a) Simulated time-sequence of critical current changes for an experiment with $N=10^{4}$ total qubit state measurements taken at intervals of $t_{Z}=1 \mathrm{~ms}$. (b) Corresponding $1 / f$ frequency spectrum.

This procedure is equivalent to sampling real and imaginary components of the critical current fluctuations from Gaussian distributions centered at zero magnitude, thus ensuring that the generated noise is Gaussian. The actual critical current fluctuations of the junction may not be strictly Gaussian if interactions between the charged traps are present, but the assumption of Gaussian statistics should give a good representation of the noise. The relevant frequency range is from $f_{\max }=1 / t_{Z}$, set by the single-shot measurement time, to $f_{\min }=1 / N t_{Z}$, where $N t_{Z}$ is the total duration of the experiment. As an example, consider an experiment in which $t_{Z}=1 \mathrm{~ms}$, $N_{Z}=100$, and $N_{t}=100$. We generate $N=10^{4}$ time sequence points over the period $N t_{z}=10 \mathrm{~s}$. We choose a representative qubit with a junction of critical current $I_{0}=1 \mu \mathrm{A}$ and area $A=0.01 \mu \mathrm{m}^{2}$. At $T=100 \mathrm{mK}$, the universal $1 / f$ noise spectral density from Eq.(19) yields $S_{I_{0}}(1 \mathrm{~Hz})=8.16 \times 10^{-24} \mathrm{~A}^{2} \mathrm{~Hz}^{-1}$, corresponding to a root-mean-square fractional change in the critical current of about $10^{-5}$ over the bandwidth from $10^{-1}$ to $10^{3}$ Hz. Figure 9 (a) shows a typical time trace simulated with these parameters. The enhanced low frequency components present in the $1 / f$ spectrum are evident in the fluctuation spectrum.

To simulate the observed coherent oscillations, we insert such a noise time-sequence of the required duration into Eqs. (21) and (22). In Fig. 10, we show the probability amplitude $\langle Z\rangle$ calculated for $N_{t}=1000$ time delay points, each averaged over $N_{Z}=3000$ qubit state 
measurements (thus, $N=3 \times 10^{6}$ ) acquired by sampling methods $\mathrm{A}$ and $\mathrm{B}$. We assume the qubit parameters $I_{0}=1 \mu \mathrm{A}, A=0.01 \mu \mathrm{m}^{2}, \Omega / 2 \pi=1 \mathrm{GHz}$ and $\Lambda=100$, with $T=100 \mathrm{mK}$. The optimum sampling rate is larger than the Nyquist frequency so that the characteristic qubit oscillation frequency can be determined, and incommensurate with the oscillation period of the qubit, so that the envelope of the oscillations is fully delineated and not aliased. In this case, we arbitrarily choose the sampling frequency to be the irrational number $(1+\phi) \Omega / 2 \pi \approx 2.618 \mathrm{GHz}$, where $\phi=(1+\sqrt{5}) / 2 \approx 1.618$ is the Golden mean. Thus, $t_{Z}=0.382 \mathrm{~ns}$. The envelope function is calculated by demodulating the oscillations via convolution of the averaged probability amplitudes with the Gaussian filter kernel

$$
K(t)=\left(\frac{1}{2 \pi \sigma^{2}}\right)^{1 / 2} \exp \left(-t^{2} / 2 \sigma^{2}\right),
$$

where $\sigma$ is chosen to be the sampling period $t_{Z}$.

The oscillation amplitude of the qubit state is found to decay with a Gaussian envelope function

$$
\langle Z\rangle_{\text {env }} \sim \exp \left(-t^{2} / 2 \tau_{\phi}^{2}\right),
$$

where $\tau_{\phi}$ is a characteristic dephasing time. This form arises from the frequency modulation of the qubit by the critical current fluctuations, in contrast to an exponential decay induced by dissipative processes. We note that for long delay times the envelope does not vanish but instead saturates to a noise floor level that corresponds to uniform randomization of the oscillation phase by the critical current fluctuations. The noise floor is $Z_{\text {noise }} \sim$ $N_{Z}^{-1 / 2}$ for both Methods A and B. Particularly for small $N_{Z}$, it is necessary to account for the noise floor to make an accurate determination of $\tau_{\phi}$. We do this by fitting to

$$
\langle Z\rangle_{\text {env }} \sim Z_{\text {noise }}+\left(1-Z_{\text {noise }}\right) \exp \left(-t^{2} / 2 \tau_{\phi}^{2}\right) .
$$

Both the dephasing times and the scatter in the amplitude envelope are different for the two methods. Method A gives a longer dephasing time than Method B, in this case by about $30 \%$. This occurs because all of the qubit state measurements at a particular delay time for Method A are acquired in a time interval $N_{Z} t_{Z}$, rather than over the entire experiment duration $N t_{Z}$ as in Method B. Thus, the number of decades of $1 / f$ noise that affect the qubit dynamics in Method $\mathrm{A}$ is $\log \left(N_{Z}\right)=3$, compared to Method B which samples $\log (N)=6$ decades. The scatter in the simulated data is also greater for Method A because the low frequency variation of the tunneling frequency is not averaged out. The origin of this scatter can be best understood by choosing junction and measurement parameters for which $\tau_{\phi}$ and $T_{o s c}$ are comparable so that the coherent oscillations and the amplitude decay can be resolved simultaneously. In Fig. 11] we show the probability amplitude for the same qubit parameters but with a substantially increased level of critical current
FIG. 10: note: figure attached Probability envelopes determined by simulations using measurement Methods A and B for a qubit with $I_{0}=1 \mu \mathrm{A}, S_{I_{0}}(1 \mathrm{~Hz})=8.16 \times 10^{-24} \mathrm{~A}^{2} \mathrm{~Hz}^{-1}$, $A=0.01 \mu \mathrm{m}^{2}, \Lambda=100$, and $\Omega / 2 \pi=1 \mathrm{GHz}$. The structure visible in the Method B plot arises from periodic sampling of the oscillations and is evidence of the increased effective averaging relative to Method $\mathrm{A}$.

FIG. 11: note: figure attached Simulated probability oscillations with large critical current fluctuations for measurement Methods A and B. Qubit parameters as in Fig. [10] except $S_{I_{0}}(1 \mathrm{~Hz})=1.39 \times 10^{-20} \mathrm{~A}^{2} \mathrm{~Hz}^{-1}$

fluctuations, approximately 40 times larger in amplitude, calculated for $N_{t}=200$. Here, the discrete oscillations are clear for Method B but quite distorted for Method A. The dephasing time for Method A is again longer, in this case by about $22 \%$.

Because of the low frequency divergence of $1 / f$ noise, the variance in the measured dephasing time is substantial, and it is necessary to carry out a series of experimental runs to determine the dephasing time accurately for a given set of junction and measurement parameters. The spread in dephasing times can be seen in Fig. 12 in which we plot distributions of the dephasing times obtained by Methods A and B for the qubit parameters used in Fig. [10] and for different numbers of flux measurements. For any value of $N$, the mean dephasing time is larger for Method A than for Method B, as expected since fewer decades of $1 / \mathrm{f}$ noise affect the qubit; the standard deviations are larger for Method B.

With a series of such simulations for different junction and qubit parameters, it is straightforward to establish that $\tau_{\phi}$ is proportional to $I_{0}$ and inversely proportional to $\Omega, \Lambda$, and $S_{I_{0}}^{1 / 2}(1 \mathrm{~Hz})$. The dependence of $\tau_{\phi}$ on the number of measurements, which sets the range of $1 / f$ noise that is effective in dephasing the qubit, can be found by carrying out the simulations for different measurement parameters $N_{t}$ and $N_{Z}$, as shown in Fig. 12. The mean dephasing times for a series of simulations with the same parameters described above are shown in Fig. 13. As discussed above, Method A gives longer times than Method B for all values of $N$. We find that the dephasing time $\tau_{\phi}$ for Method A decreases as a weak power-law of $N$, which is expected since the frequency range of the $1 / f$ noise increases for larger $N_{Z}$. For Method $\mathrm{B}, \tau_{\phi}$ is nearly constant, changing by only a few percent over 3 orders of magnitude in $N$. This insensitivity likely arises because the increased frequency range of the noise for larger $N$ (which should suppress the the dephasing time) is compensated by the increased averaging which smoothes the fluctuations. For large $N, \tau_{\phi}$ for Method B agrees well with the analytical result obtained by Martinis, et al. 12 differing only by a numerical factor of order unity, but deviates substantially at lower $N$.

Using our empirical expression for $S_{I_{0}}(f)$, Eq. (19), 
FIG. 12: note: figure attached Distributions of dephasing times $\tau_{\phi}$ calculated by Method A (open symbols) and Method $\mathrm{B}$ (closed symbols) for different number of flux measurement points $N=3 \times 10^{4}$ (squares), $3 \times 10^{5}$ (triangles), and $3 \times 10^{6}$ (circles). Each distribution includes 1000 simulations of the coherent oscillations accumulated into bins of width 2 ns. Qubit parameters are as in Fig. 10]

and taking the number of qubit measurements in a typical experiment to be $N=10^{6}$, we find

$$
\tau_{\phi}^{A}(\mu \mathrm{s}) \approx 20 A^{1 / 2}(\mu \mathrm{m}) / \Lambda(\Omega / 2 \pi)(\mathrm{GHz}) T(\mathrm{~K})
$$

for sampling by Method A and

$$
\tau_{\phi}^{B}(\mu \mathrm{s}) \approx 15 A^{1 / 2}(\mu \mathrm{m}) / \Lambda(\Omega / 2 \pi)(\mathrm{GHz}) T(\mathrm{~K})
$$

for Method B.

¿From these results, we estimate the values of $\tau_{\phi}$ and $\Omega \tau_{\phi} / 2 \pi$ predicted for each of the qubit schemes described in Sec. III, using the device parameters reported in the experiments and assuming sampling by Method B with $N=10^{6}$. We have set $T=100 \mathrm{mK}$ and assumed explicitly that the $T^{2}$ dependence of $S_{I_{0}}(f)$ extends to this temperature. These results are listed in Table II. For comparison, we also list the measured dephasing times and the temperatures at which the experiments were performed. Our estimated dephasing times range between $0.8 \mu \mathrm{s}$ and $12 \mu \mathrm{s}$, with the longer times corresponding to the qubit schemes with larger area junctions. Such times would allow for several thousand oscillations of the quantum state, making possible various quantum computing operations. However, with the exception of quantronium, the measured dephasing times are orders of magnitude shorter than our estimated values, indicating that other sources of decoherence are dominant. In the quantronium experiments, the isolation obtained by operating at the optimal working point, described in Section III.E, enhances the coherence time nearly to the value where our estimates (at $100 \mathrm{mK}$ ) predict critical current fluctuations would have a noticeable effect; however, $S_{I_{0}}$ may be substantially smaller at the experimental temperature of $15 \mathrm{mK}$.

\section{CONCLUSIONS}

Despite ongoing studies over more than two decades, the origin of $1 / f$ noise in the critical current of Josephson junctions is still not fully understood. Although there is

\section{FIG. 13: note: figure attached}

Variation of the dephasing time $\tau_{\phi}$ with the number of qubit state measurements $N$ for Methods A and B. Each point corresponds to the mean value of $\tau_{\phi}$ from 50 simulations of the oscillation decay envelope. Qubit and noise parameters as in Fig. 10 strong evidence that the noise derives from a superposition of random telegraph signals produced by charge trapping and untrapping processes, the origin of the $T^{2}$ dependence observed by Wellstood ${ }^{25}$ remains puzzling. This temperature dependence can be explained within the framework of a two-well potential in which the two barrier heights are independent random variables, provided one assumes thermally-activated processes rather than the tunneling processes one might expect. Furthermore, the absence of a temperature dependence of the form $\exp \left(-\Delta / k_{B} T\right)$ at low temperatures is difficult to understand in a picture in which the trap exchanges single electrons with superconducting electrodes. Clearly more work is required to understand this behavior. We found that the measured spectral density of the $1 / f$ noise in the critical current of junctions with different materials and a wide range of areas and critical currents scales surprisingly well as $\left[144\left(I_{0} / \mu \mathrm{A}\right)^{2} /\left(A / \mu \mathrm{m}^{2}\right)\right](\mathrm{pA})^{2} / \mathrm{Hz}$ at 4.2 $\mathrm{K}$. Based solely on the results of Wellstood we have chosen to scale this number with $(T / 4.2 \mathrm{~K})^{2}$ to predict the $1 / f$ noise at $100 \mathrm{mK}$. How well this scaling remains valid as more junctions are investigated and whether the $T^{2}$ dependence holds down to (say) $10 \mathrm{mK}$ are questions that should be addressed with some urgency. These measurements must of necessity be made with a SQUID amplifier; the use of submicron junctions with relatively high critical currents should enhance the magnitude of the noise and make its observation more straightforward.

For four different qubits we calculated the parametric effect of small changes in the critical current $I_{0}$ on the energy separation $\hbar \Omega$ at the operating point. Using the normalized parameter $\Lambda=\left|I_{0} d \Omega / \Omega d I_{0}\right|$ and the extrapolated magnitude of the $1 / f$ noise we investigate dephasing in these qubits at $0.1 \mathrm{~K}$. In agreement with the treatment of Martinis et al. 12 we find that the sources of decoherence accumulate as $t^{2}$, so that the decoherence is not interpretable as a rate. Rather, the frequency is different each time a measurement is made. In all cases where $\tau_{\phi}$ has been measured, the calculated values due to critical current $1 / f$ noise are greater than the measured values. Furthermore, if the $T^{2}$ dependence of the $1 / f$ noise does continue at temperatures down to (say) $10 \mathrm{mK}$, the predicted decoherence time, which scales as $1 / T$, will become an order of magnitude longer at this temperature. Nonetheless, although critical current $1 / f$ noise appears not to be the limiting source of decoherence in experiments conducted to date, ultimately this mechanism will present an upper bound on $\tau_{\phi}$.

Although the level of $1 / f$ noise is remarkably constant for existing junction technologies, there may be alternative schemes for growing the tunnel barrier which reduce the number of charge traps in the barrier, and hence reduce the noise. We note also that even in the presence of low frequency noise, the use of various pulse sequences, such as spin echoes, $, 4,11,30$ or bang-bang pulses 31 can significantly reduce its effects.

Finally, in the case of flux qubits this formulation could be extended to the effects of $1 / f$ flux noise originating 
TABLE II: Estimated dephasing times at $100 \mathrm{mK}$ due to $1 / f$ noise in $I_{0}$ for various qubit schemes. Measured dephasing times and experimental temperatures are included where measurements exist. For the one-junction flux qubit columns, values of $\Omega / 2 \pi$ were calculated as described in the text. All other values of $\Omega / 2 \pi$ were taken from corresponding experiments. Values of $\Lambda$ for each qubit scheme were calculated as described in Sec. III.

\begin{tabular}{cccccc}
\hline Parameter & $\begin{array}{c}\text { 1-junction } \\
\text { flux qubit } \\
\text { (ground state) }\end{array}$ & $\begin{array}{c}\text { 1-junction } \\
\text { flux qubit } \\
\text { (excited state) }\end{array}$ & $\begin{array}{c}\text { 3-junction } \\
\text { flux qubit }\end{array}$ & $\begin{array}{c}\text { single } \\
\text { junction }{ }^{\underline{*}}\end{array}$ & quantronium $^{7}$ \\
\hline \hline$I_{0}(\mu \mathrm{A})$ & 1.46 & 1.46 & 0.5 & 21.1 & 0.018 \\
$A\left(\mu \mathrm{m}^{2}\right)$ & 2.0 & 2.0 & 0.05 & 100 & 0.02 \\
$\Lambda$ & 40.6 & 71.5 & 12.3 & 16 & 0.7 \\
$\Omega / 2 \pi(\mathrm{GHz})$ & 3.4 & 0.59 & 3.4 & 6.9 & 16.5 \\
calc $\tau_{\phi}(\mu \mathrm{s})(100 \mathrm{mK})$ & 1.5 & 5.1 & 0.8 & 14 & 1.8 \\
meas $\tau_{\phi}(\mu \mathrm{s})(T / \mathrm{mK})$ & - & - & $0.02(25)$ & $0.01(25)$ & $0.50(15)$ \\
calc $\Omega \tau_{\phi} / 2 \pi(100 \mathrm{mK})$ & 5100 & 3000 & 2700 & 97000 & 30000 \\
meas $\Omega \tau_{\phi} / 2 \pi(T / \mathrm{mK})$ & - & - & $68(25)$ & $69(25)$ & $8000(15)$ \\
\hline
\end{tabular}

from either magnetic vortex motion or current noise in the current supply by calculating the quantity $d \Omega / d \Phi$.

\section{Acknowledgments}

We thank Tony Leggett, John Martinis, Michael Weissman, Fred Wellstood, and Frank Wilhelm for useful dis- cussions. This work was supported in part by the Air Force Office of Scientific Research under Grant F49620-02-1-0295, the Army Research Office under Grant DAAD-19-02-1-0187, and the National Science Foundation under Grants EIA-020-5641 and EIA-01-21568. DVH thanks the Miller Institute at the University of California, Berkeley, for Fellowship support.
1 J. R. Friedman, V. Patel, W. Chen, S. K. Tolpygo, and J. E. Lukens, Nature 406, 43 (2000).

2 A. Leggett, Les Houches, Session XLVI - Chance and Matter (Elsevier, Amsterdam, 1987), pp. 395-506.

${ }^{3}$ C. H. van der Wal, A. C. J. T. Haar, F. K. Wilhelm, R. N. Schouten, C. J. P. M. Harmans, T. P. Orlando, S. Llyod, and J. E. Mooij, Science 290, 773 (2000).

4 I. Chiorescu, Y. Nakamura, C. J. P. M. Harmans, and J. E. Mooij, Science 299, 1869 (2003).

${ }^{5}$ Y. Nakamura, Y. A. Pashkin, and J. S. Tsai, Nature 398, 786 (1999).

6 Y. A. Pashkin, T. Yamamoto, O. Astafiev, Y. Nakamura, D. V. Averin, and J. S. Tsai, Nature 421, 823 (2003).

7 D. Vion, A. Aassime, A. Cottet, P. Joyez, H. Pothier, C. Urbina, D. Esteve, and M. H. Devoret, Science 296, 886 (2002).

8 A. Cottet, D. Vion, A. Aassime, P. Joyez, D. Esteve, and M. H. Devoret, Physica C 367, 197 (2002).

9 J. M. Martinis, S. Nam, J. Aumentado, and C. Urbina, Phys. Rev. Lett. 89, 117901 (2002).

10 Y. Yu, S. Han, X. Chu, S.-I. Chu, and Z. Wang, Science 296, 889 (2002).

11 Y. Nakamura, Y. A. Pashkin, T. Yamamoto, and J. S. Tsai, Phys. Rev. Lett. 88, 047901 (2002).

12 J. M. Martinis, S. Nam, J. Aumentado, K. M. Lang, and C. Urbina, Phys. Rev. B 67, 94510 (2003).

13 E. Paladino, L. Faoro, G. Falci, and R. Fazio, Phys. Rev. Lett. 88, 228304 (2002).

14 E. Dantsker, S. Tanaka, P.-R. Nilsson, R. Kleiner, and
J. Clarke, Appl. Phys. Lett. 69, 4099 (1996).

15 J. Clem, unpublished.

16 R. T. Wakai and D. J. V. Harlingen, Appl. Phys. Lett. 49, 593 (1986).

17 C. Rogers and R. Buhrman, Phys. Rev. Lett. 53, 1272 (1984).

18 C. Rogers and R. Buhrman, Phys. Rev. Lett. 55, 859 (1985)

19 R. T. Wakai and D. J. V. Harlingen, Phys. Rev. Lett. 58, 1687 (1987).

20 P. Dutta and P. M. Horn, Rev. Mod. Phys. 53, 497 (1981).

${ }^{21}$ M. B. Weissman, Rev. Mod. Phys. 60, 537 (1988).

22 A. Garg, Am. J. Phys. 68, 430 (2000).

23 D. V. Averin, J. R. Friedman, and J. E. Lukens, Phys. Rev. B 62, 11802 (2000).

24 T. P. Orlando, J. E. Mooij, L. Tian, C. H. van der Wal, L. S. Levitov, S. Lloyd, and J. J. Mazo, Phys. Rev. B 60, 15398 (1999).

${ }^{25}$ F. Wellstood, Ph.D. thesis, University of California, Berkeley (1988).

26 M. Kenyon, C. Lobb, and F. Wellstood, J. Appl. Phys. 88, 6536 (2000).

27 B. Savo, F. C. Wellstood, and J. Clarke, Appl. Phys. Lett. 50, 1757 (1987).

28 V. Foglietti, W. F. Gallagher, M. B. Ketchen, A. W. Kleinsasser, R. H. Koch, S. I. Raider, and R. L. Sandstrom, Appl. Phys. Lett. 49, 1393 (1986).

29 R. H. Koch, D. J. V. Harlingen, and J. Clarke, Phys. Rev. B 26, 74 (1982). 
${ }^{30}$ E. L. Hahn, Phys. Rev. 80, 580 (1950).

31 H. Gutmann, F. K. Wilhelm, W. M. Kaminsky, and

S. Lloyd, \cond-mat \0308107 (2003). 
This figure "figures-1_2.png" is available in "png" format from: http://arxiv.org/ps/cond-mat/0404307v1 
This figure "figures-3.png" is available in "png" format from: http://arxiv.org/ps/cond-mat/0404307v1 
This figure "figures-4.png" is available in "png" format from: http://arxiv.org/ps/cond-mat/0404307v1 
This figure "figures-5.png" is available in "png" format from: http://arxiv.org/ps/cond-mat/0404307v1 
This figure "figures-6.png" is available in "png" format from: http://arxiv.org/ps/cond-mat/0404307v1 
This figure "figures-7.png" is available in "png" format from: http://arxiv.org/ps/cond-mat/0404307v1 
This figure "figures-8.png" is available in "png" format from: http://arxiv.org/ps/cond-mat/0404307v1 
This figure "figures-9.png" is available in "png" format from: http://arxiv.org/ps/cond-mat/0404307v1 
This figure "figures-10.png" is available in "png" format from: http://arxiv.org/ps/cond-mat/0404307v1 
This figure "figures-11.png" is available in "png" format from: http://arxiv.org/ps/cond-mat/0404307v1 
This figure "figures-12.png" is available in "png" format from: http://arxiv.org/ps/cond-mat/0404307v1 
This figure "figures-13.png" is available in "png" format from: http://arxiv.org/ps/cond-mat/0404307v1 